\title{
Catálogos de litotipos empleados en el Patrimonio Inmueble Andaluz
}

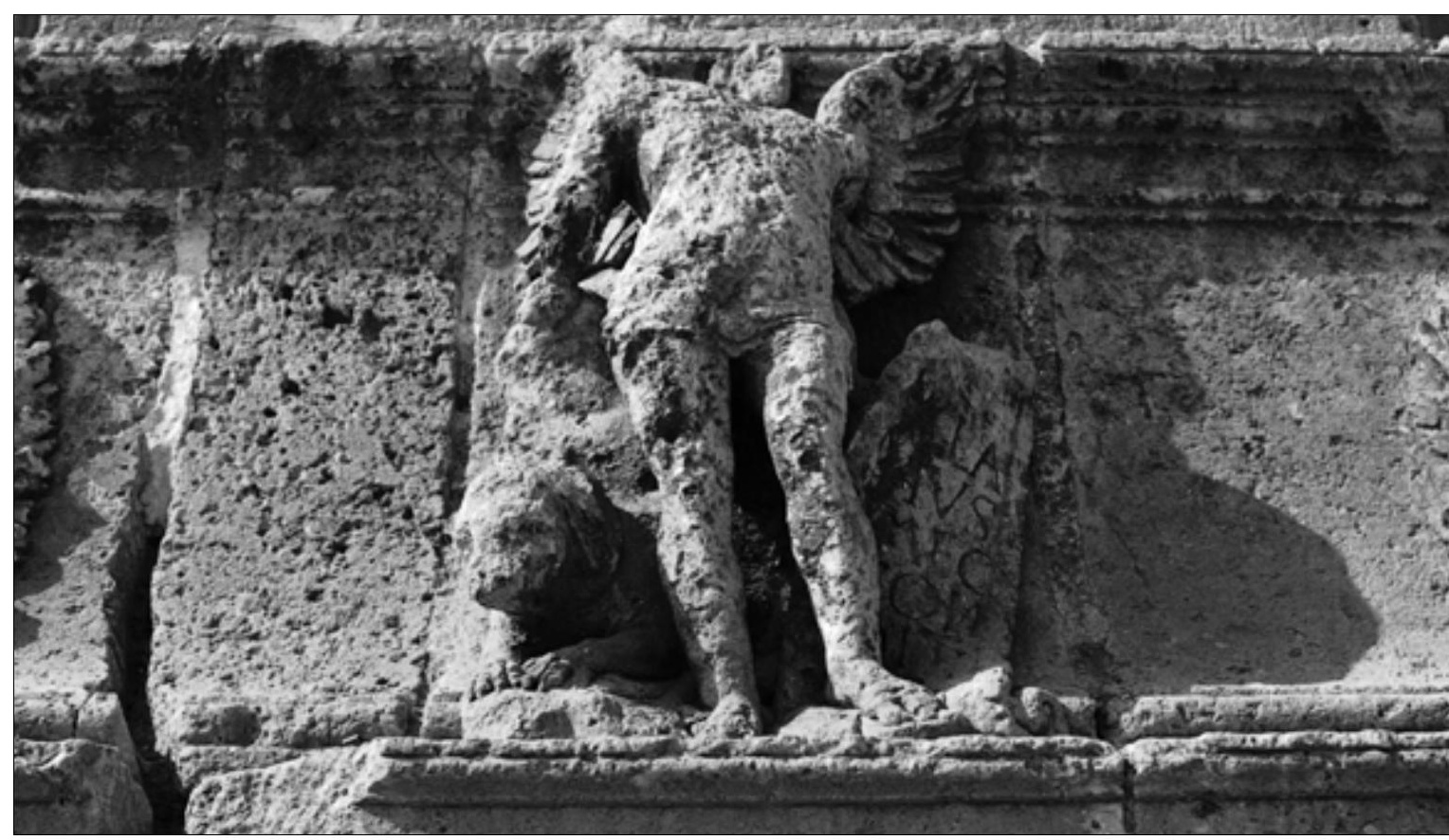

Rosario Villegas Sánchez

Departamento de análisis Instituto Andaluz del Patrimonio Histórico a piedra es un material empleado con profusión en el Patrimonio Inmueble, en general, y en nuestra comunidad en particular. El estado de alteración de los edificios depende en gran parte de las características de los materiales utilizados, lo que se denomina factores de alteración intrínsecos. Cuando se acomete una intervención de conservación-restauración, es imprescindible conocer los materiales sobre los que se va a actuar, sus propiedades, las alteraciones que presentan y su posible respuesta a los tratamientos que se vayan a realizar.

En un edificio se encuentran dos grupos de materiales inorgánicos diferentes; aquéllos manufacturados: ladrillos y otras cerámicas, morteros, revestimientos, etc, y los no preparados, la piedra. En el caso de los primeros, su composición y, por tanto, sus características pueden variar apreciablemente de un edificio a otro e incluso en distintas zonas de un mismo edificio, por lo que deben ser estudiados específicamente en cada caso.

Por el contrario, la piedra de una determinada cantera mantiene similares características (dentro de ciertos límites) en todos los edificios donde se haya utilizado; es- 
tas características contribuyen a explicar, en gran medida, su comportamiento y el estado de conservación y los indicadores de alteración que presenta. Por ello, sí que es posible utilizar los datos obtenidos en un edificio o en la propia cantera como información de partida para aquellos otros donde se encuentren los mismos litotipos.

Diversos equipos de investigación, especialmente de las Universidades y más recientemente del I.A.P.H., se vienen dedicando desde hace bastantes años, a estudiar los distintos materiales pétreos empleados en el Patrimonio Inmueble andaluz, cubriendo entre todos ellos prácticamente todas las provincias de la Comunidad y una gran parte de los monumentos existentes.

Sin embargo, los resultados de estas investigaciones se difunden en círculos cientíicos relativamente restringidos y muchas veces no llegan a los que deberían ser sus usuarios principales, los arquitectos y restauradores encargados de llevar a cabo proyectos de conservación-restauración de los edificios. De esta forma, cuando se va a llevar a cabo la intervención, en ocasiones se duplican los estudios analíticos de los materiales y otras veces no se llegan a realizar estos análisis.

Para intentar paliar, al menos en parte, esta situación, el I.A.P.H. ha tomado la iniciativa de recopilar y publicar todos los datos disponibles de materiales pétreos empleados en nuestra comunidad y procedentes, la mayoría de ellos, también de canteras de la región. Se ha elaborado una ficha, lo más amplia posible, en la que se recogen las características más significativas de cada litotipo, además de su procedencia y algunos ejemplos de edificios donde se haya utilizado. En la primera parte se ha utilizado la propuesta de descripción petrográfica que aparece en este mismo número; los métodos usados para la determinación de las restantes características se irán publicando dentro del Programa de Normalización de Estudios Previos que tiene en marcha el I.A.P.H.

En esta fase han participado activamente, junto con el Departamento de Análisis del Centro de Intervención, el Departamento de Mineralogía y Petrología de la Universidad de Granada y el Departamento de Ingeniería Química y Ambiental de la Universidad de Sevilla. Los materiales pétreos cuyas características se van a ir publicando periódicamente son fundamentalmente los estudiados por estos tres grupos, pero nos gustaría recibir aportaciones de otros investigadores con los datos correspondientes a las piedras sobre las cuales hayan trabajado.

La ficha se estructura en forma de tablas, que pueden utilizarse para una o más variedades de un mismo litotipo. En la primera parte se recogen las características mineralógico-petrográficas y la composición química; a continuación las propiedades físicas y mecánicas; en tercer lugar el comportamiento hídrico en forma de gráficas de absorción y desorción de agua, junto con los valores numéricos. Por último, se incluyen unas consideraciones muy resumidas sobre el comportamiento del material (o de las diversas variedades), y fotografías de microscopía óptica y del aspecto macroscópico y alteraciones características de la piedra.

Los datos recogidos en las fichas que se publican en este número y posteriores proceden de trabajos de investigación y/o Tesis doctorales en los que han participado los siguientes investigadores:

\section{UNIVERSIDAD DE GRANADA}

Eduardo Sebastián Pardo

Carlos Rodríguez Navarro

Ma José de la Torre López

Olga Cazalla Vázquez

\section{UNIVERSIDAD DE SEVILLA}

José Francisco Vale Parapar

Manuel Alcalde Moreno

Miguel Angel Bello López

Juan Luis Pérez Bernal

\section{INSTITUTO ANDALUZ DEL PATRIMONIO HISTÓRICO}

Rosario Villegas Sánchez

Jesús Espinosa Gaitán

Esther Ontiveros Ortega

Francisco Gutiérrez Montero

Lourdes Martín García

Agradecemos su participación en la preparación del presente Catálogo. 

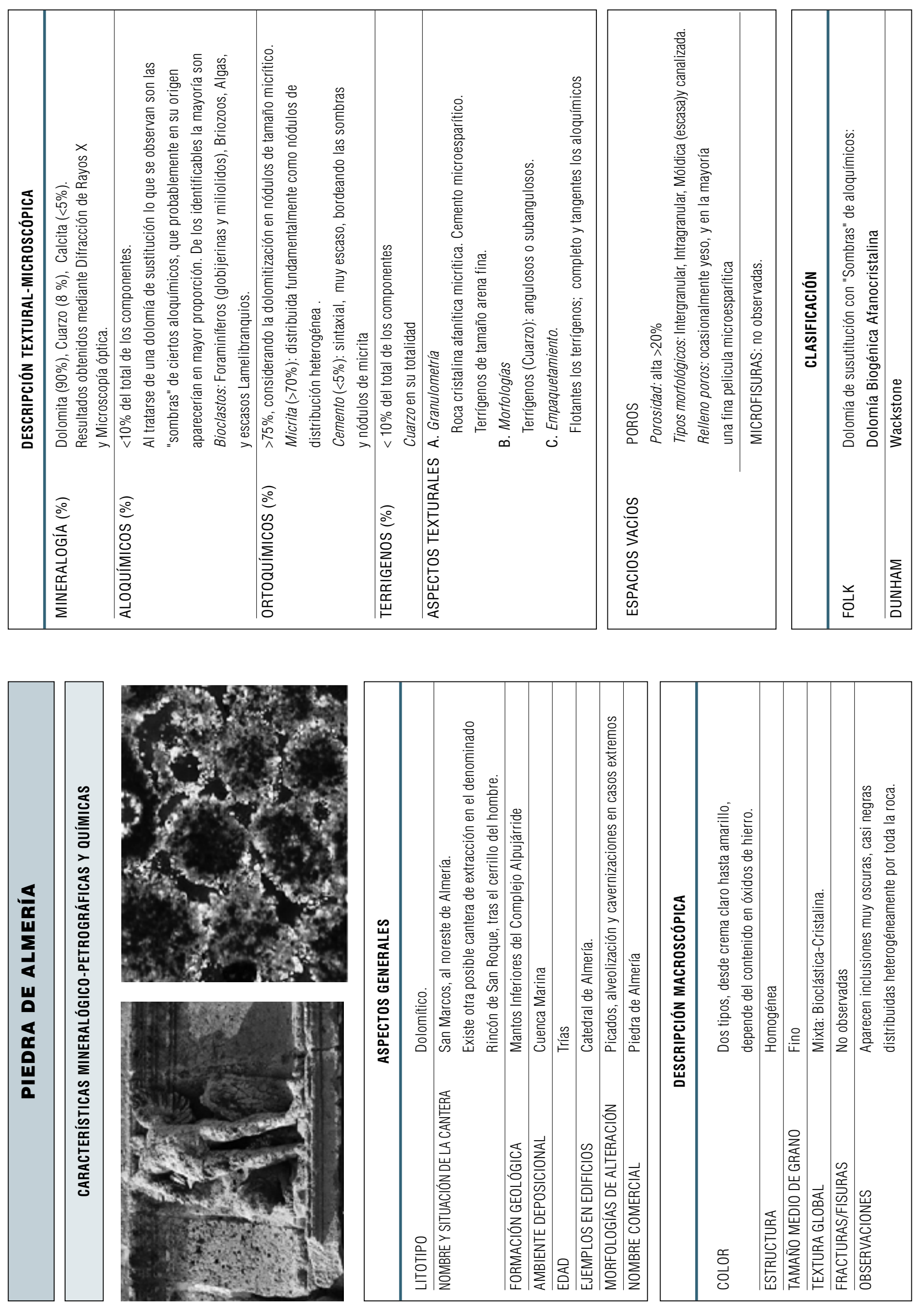

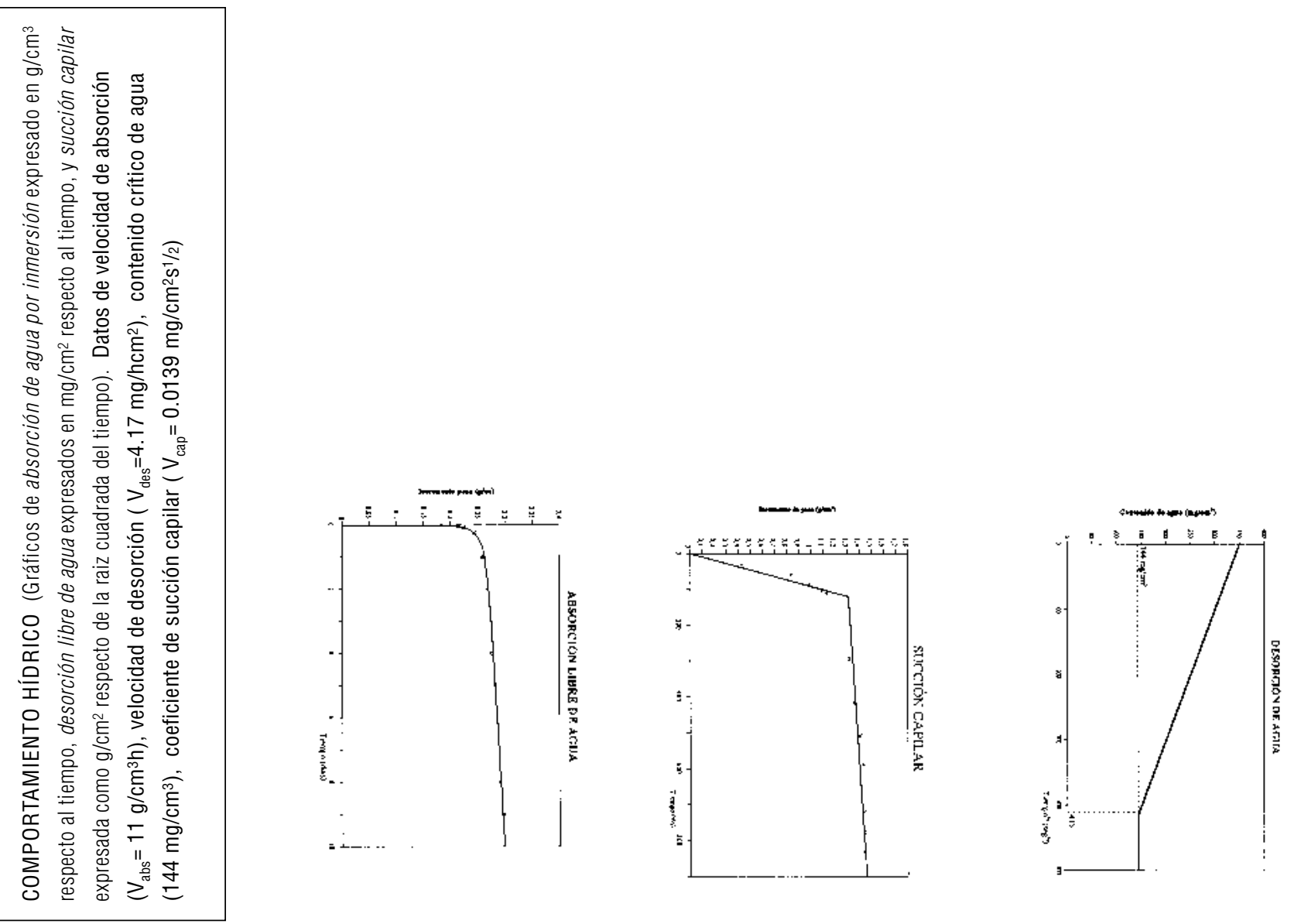

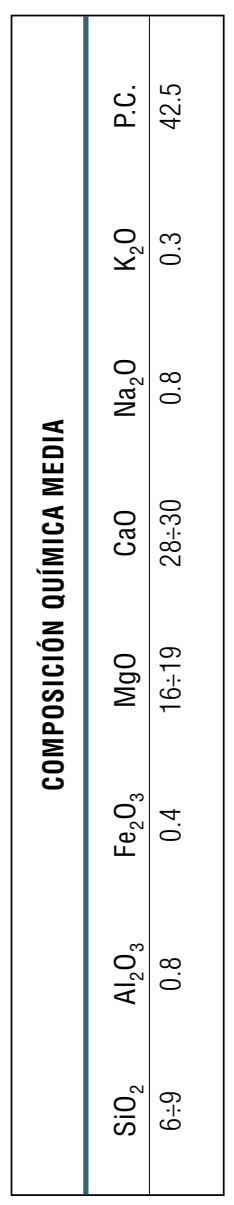

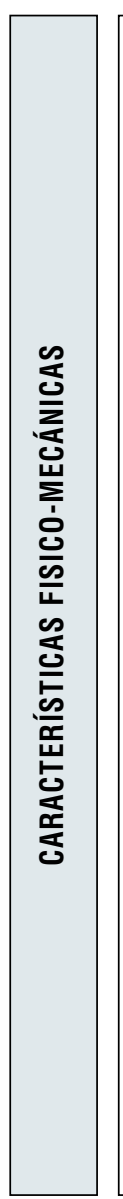

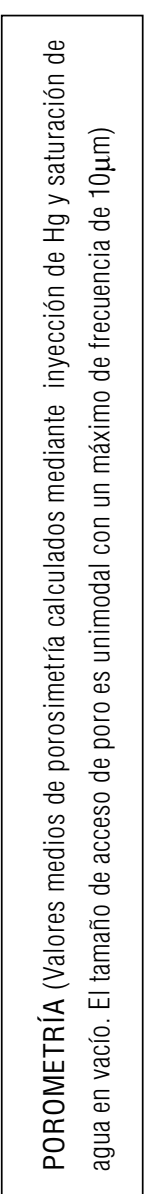
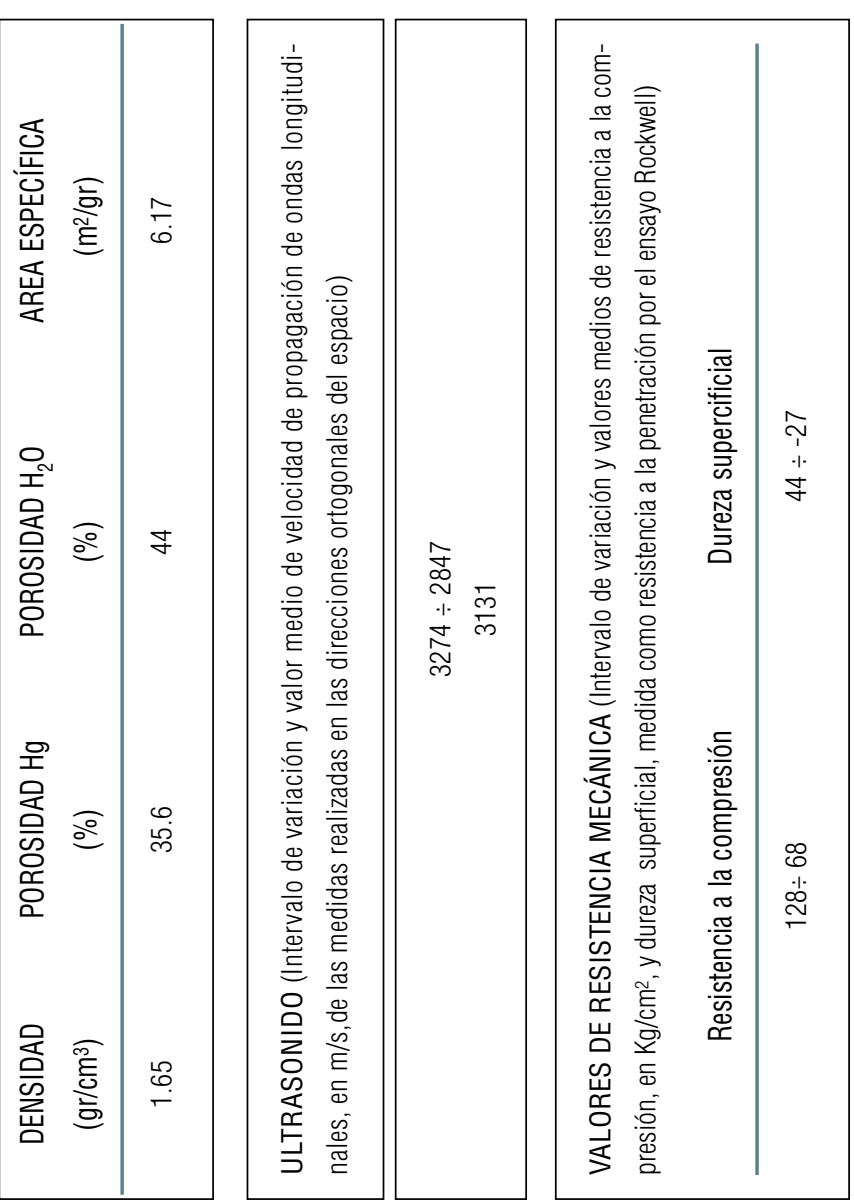

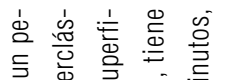

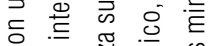

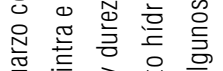

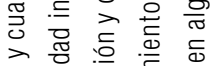

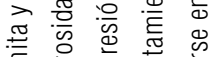

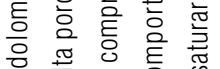
흥 栗 0

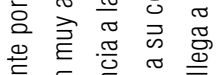
壱

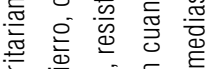

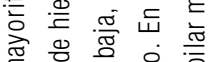

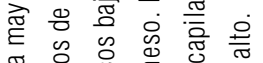

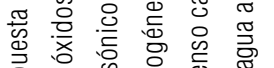

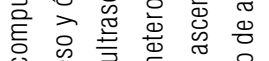
范

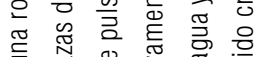

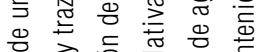

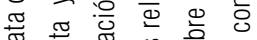
픈 늉

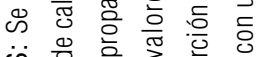

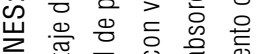
은 듕 즁

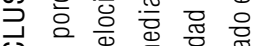

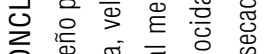

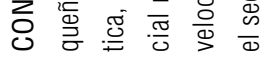



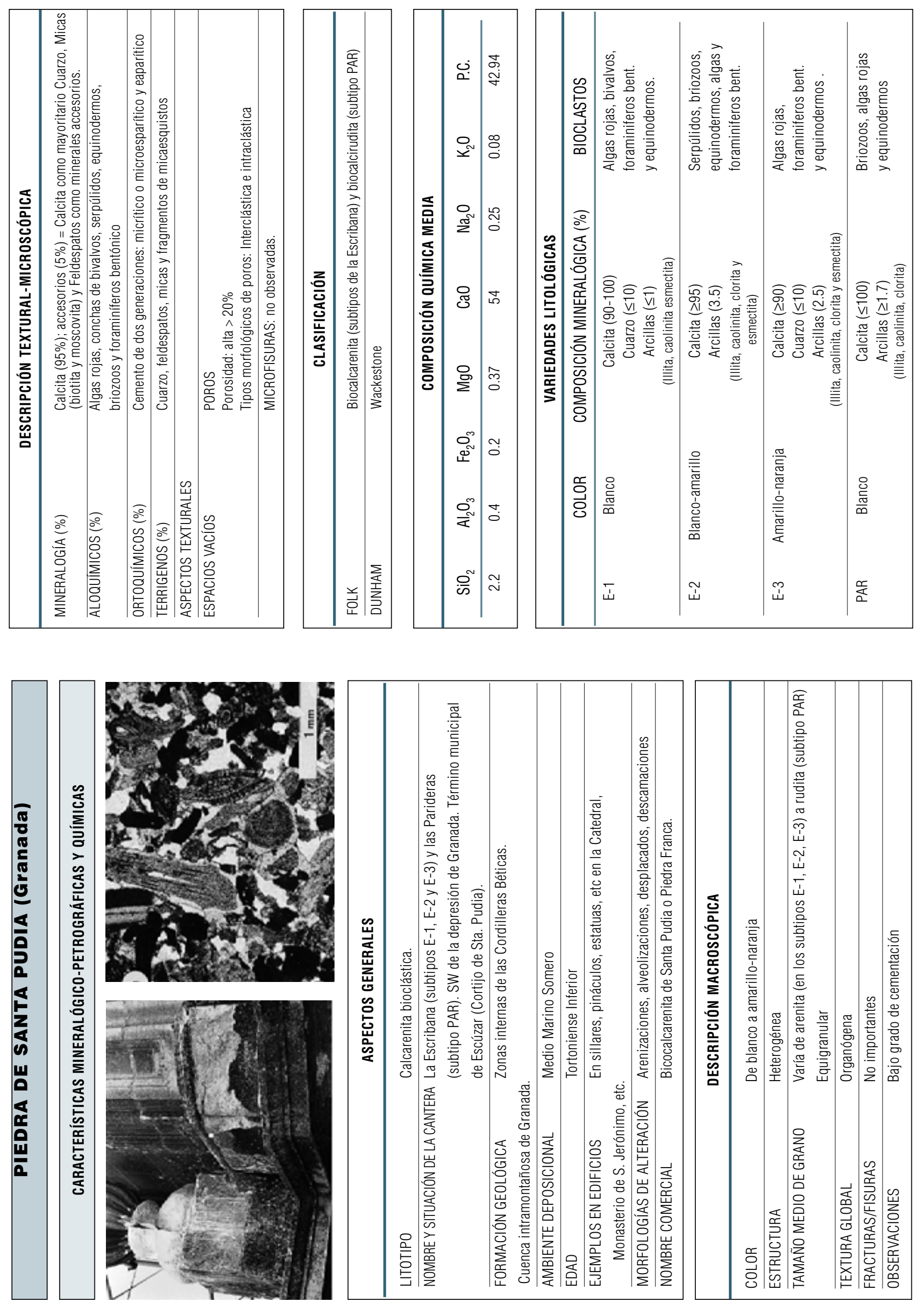


\begin{tabular}{|c|}
\hline 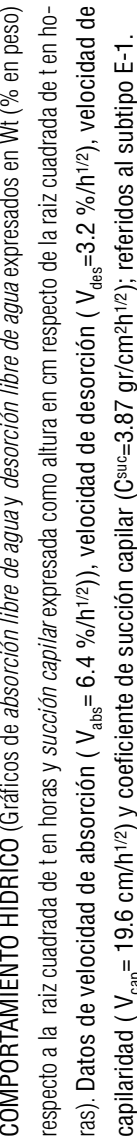 \\
\hline
\end{tabular}
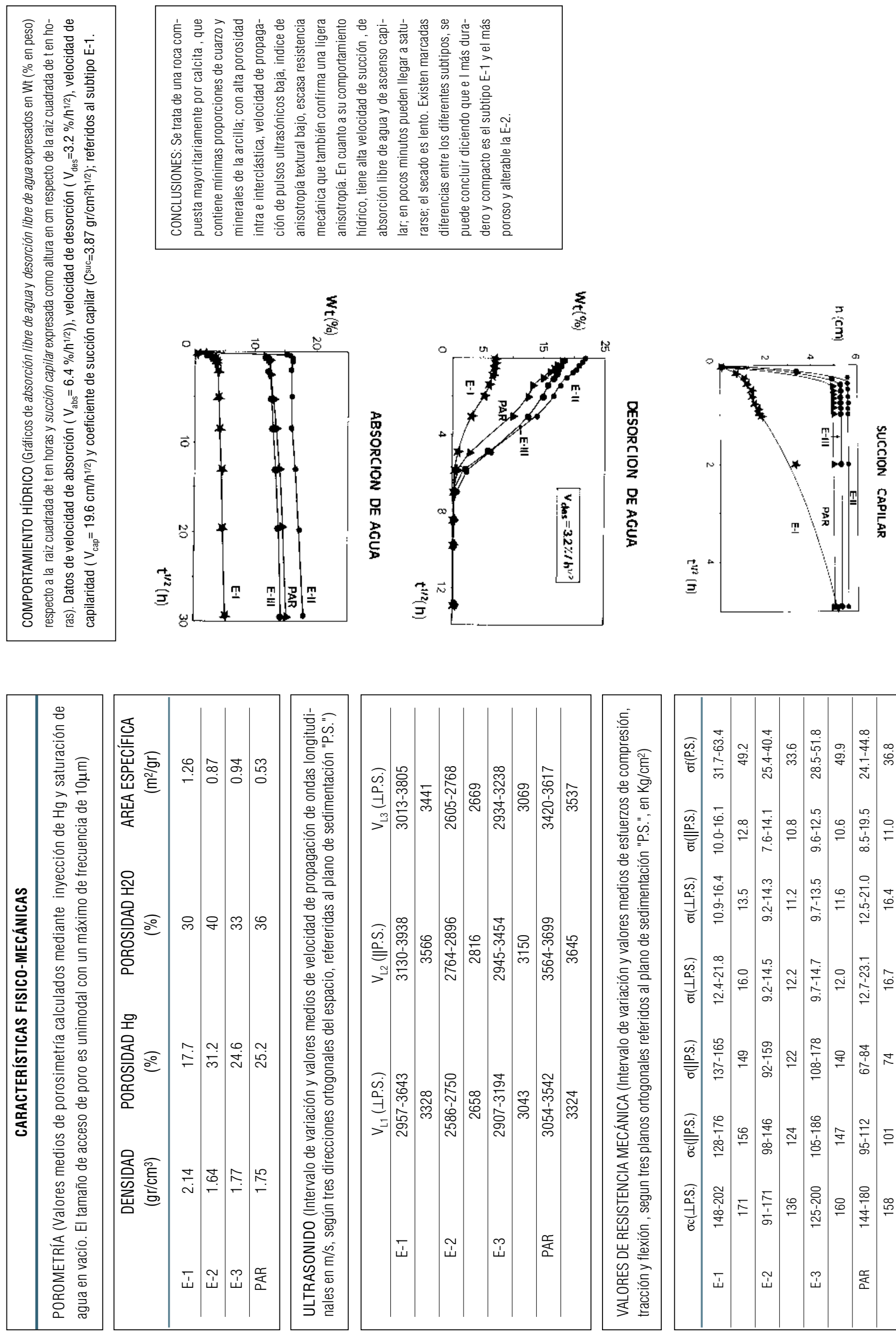

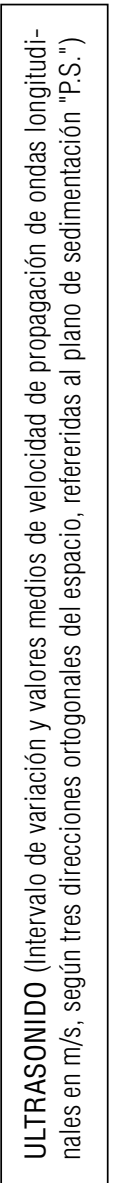
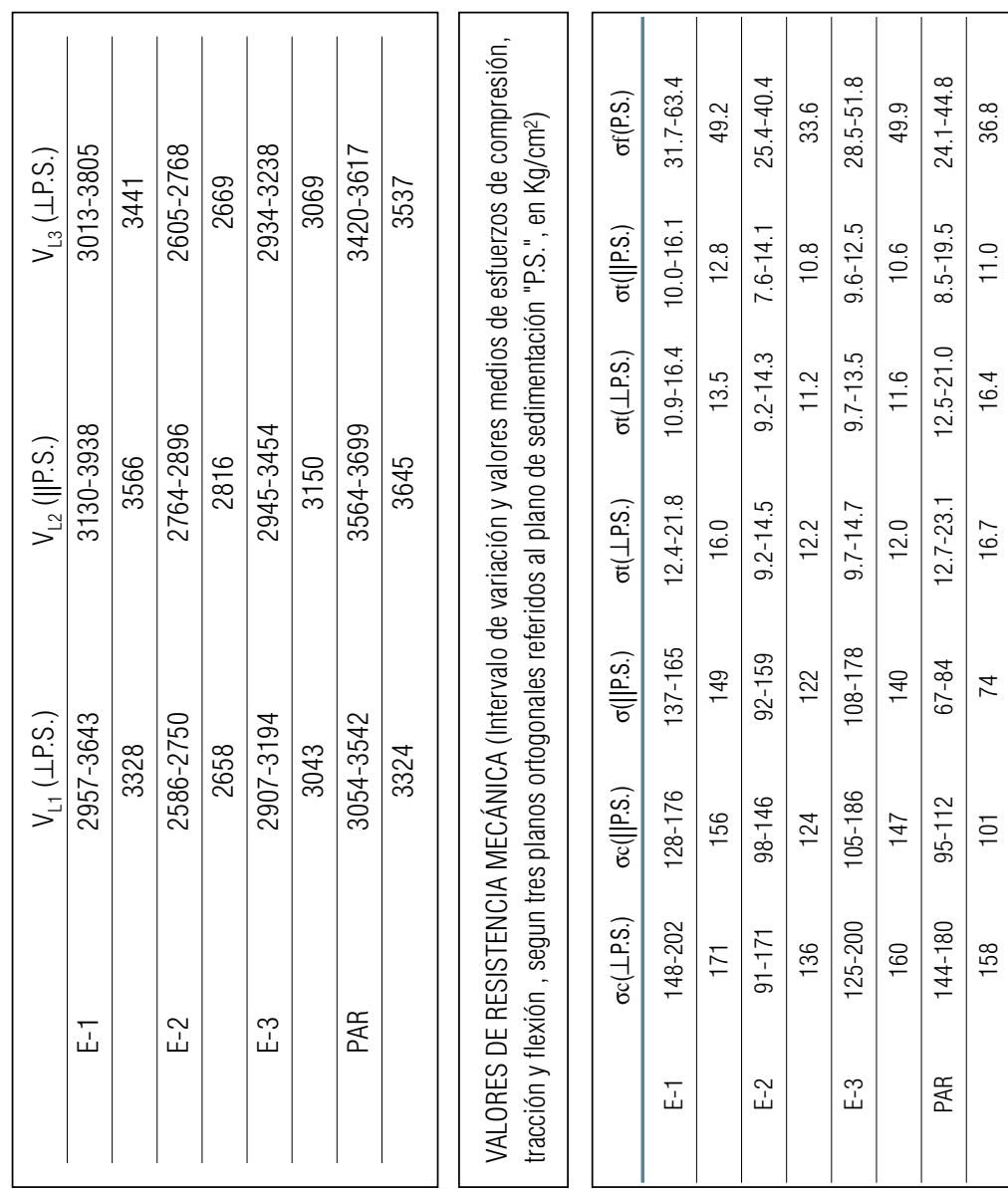

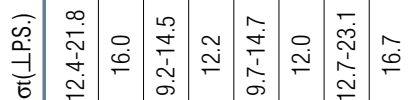

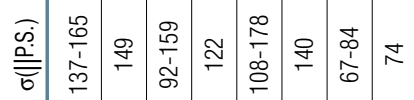

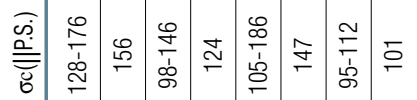

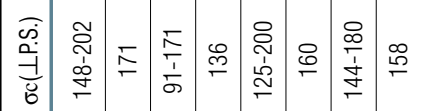

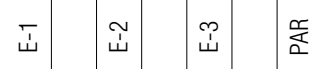

